\title{
Case Analysis of SHENXIANYUAN Financial Fraud Based on the GONE-theory
}

\author{
Ye Song-qin; Hu Si-tong; Sun Lei \\ The financial college of Jiangxi normal university \\ Nanchang, Jiangxi, China
}

\begin{abstract}
With the continuous development and improvement of China's market economy, the securities market is also facing new challenges, not only opportunities, but also huge risks. At present, it seems that there are problems such as inappropriate related party transactions in listed companies in China, and there is still a trend of further expansion. Poor performance of many corporations in the first fiscal year after they were included into National Equities Exchange and Quotations (NEEQ), seriously dampened investors' faith and enthusiasm on these NEEQ-listed companies. Based on the famous GONE theory, this paper explores the most serious fraud case of SHENXIANYUAN in NEEQ which got punished by the SFC in 2016, analyzes its full motivations of financial report fraud from the point of four factors-Greed, Need, Opportunity and Exposure, then provides solving ideas on pre-market financial statements fraud issues.
\end{abstract}

Keywords-SHENXIANYUAN; Financial fraud; NEEQ; GONE-theory

\section{INTRODUCTION}

Liaoning Shen Xian Yuan Co., Ltd. (referred to as Shen Xian Yuan) was established in 2006. In 2012, it was still in a loss state. It sold a large amount of ginseng in 2013 and turned a profit. At the end of 2014, it was listed on NEEQ, known as the first stock of "wild ginseng". However, the first annual report of Shen Xian Yuan in NEEQ reflected a sharp decline in the company's performance. It coincided with the suspension of the construction of major asset restructuring by Shen Xian Yuan. the SFC launched a case investigation on it, and issued the "notice of administrative punishment" in May 2016. The investigation, which lasted nearly one year, unveiled the veil of the first case of financial fraud in NEEQ. Relevant responsible personnel also faced administrative fines and potential claims. Shen Xian Yuan had a relatively serious negative impact on the society, which further damped investors' confidence in NEEQ ${ }^{[1]}$. This paper first analyzes the financial fraud methods of Shen Xian Yuan, and uses the theory of GONE four factors to analyze the causes of financial fraud $^{[2]}$. Based on this, it provides some solutions to the problem of financial fraud management before the NEEQlisted companies.

\section{ANALYSIS ON THE FRAUD METHODS OF SHEN XIAN YUAN FINANCIAL STATEMENTS}

According to the financial report, the total profit of Shen Xian Yuan in 2012 was negative, with a loss of 23.29 million yuan. However, the annual sales revenue increased significantly in 2013, with a profit of 115 million yuan.

Fund Project: Project "Policy Burden of State-Owned Enterprises, Transparency of Financial Information and Efficiency of Government Preferential Resource Allocation" supported by the National Social Science Foundation of China(16BGL047); Social Science Planning Project of Jiangxi Province "Government Intervention, Marketization Reform and Executive Incentives of State-Owned Enterprises” (14GL52).
The SFC investigation found that Shen Xian Yuan concealed the fact of purchasing wild ginseng in 2013, and reduced the purchase cost by 55.382 million yuan, which inflated the profit of 55.382 million yuan. At the same time, the company sold a large amount of wild ginseng to related party Liaoning Shen Xian Yuan Wine Co., Ltd. (hereinafter referred to as "Shen Xian Yuan Wine Industry"), and the SFC determined that its use of unfair price increased the profit by 73.729 million yuan. The annual profit in 2013 totaled 129.115 million yuan, accounting for $116 \%$ of the net profit after taxation of Shen Xian Yuan. In fact, it was still in deficit from 2012 to 2013, and the company's performance in 2014 slowed down significantly. Shen Xian Yuan is mainly engaged in financial fraud through two means of fraud, one is less than the cost of wild ginseng, and the other is inflated related transaction revenue, accounting for 43 percent and 57 percent, respectively.

A. Falsely reporting the number of wild ginseng mining and excavating, the ginseng is actually from the purchased ginseng

The transfer of the equity statement shows that Shen Xian Yuan began to purchase wild forests in 2007 to plant wild ginseng. As the purchased mountain forest has long grown wild ginseng, it began to sell wild ginseng in mountain forest that has been growing for 15 years. In 2013, more than 260,000 wild ginseng (including scattered ginseng) were excavated, which was later changed to claim that only 205,400 wild ginseng were excavated in 2013 in the annual report of 2015. In fact, more than half of them were purchased from outside rather than produced by themselves ${ }^{[3]}$.

\section{B. Covering the purchase of wild ginseng by paying the name} of the breeding fee

The SFC found in its investigation that Shen Xian Yuan paid 55,382,210 yuan to a certain mountain ginseng farm in 2013 after signing several mountain ginseng breeding agreements with zhong and a mountain cargo village. However, as a matter of fact, Shen Xian Yuan entrusted zhong and a mountain cargo village to help him purchase wild ginseng. Shen Xian Yuan purchased a total of 126,080 pieces of whole ginseng and $2705.19 \mathrm{~kg}$ of broken ginseng through the intermediary. The procurement cost was 55,382,210 yuan (the broken ginseng is 2,000 yuan per $\mathrm{kg}$ and a whole ginseng is about 396 yuan). The so-called breeding cost is actually the cost of buying ginseng, so the cost is underestimated. 


\section{Entering the "administrative expenses" first and then transfer to the "productive biological assets"}

Shen Xian Yuan will take the cost of acquiring wild ginseng as the ginseng breeding fee into the "administrative expenses", and then use the cost of the breeding agreement to capitalize. Shen Xian Yuan will transfer the "administrative expenses" to the "productive biological assets" subject, which directly increased the profit statement by 55,382,210 yuan. Moreover, the reduction of the capital corresponds to the increase of biological assets, so that the company's net asset valuation does not decreased, and then the inflated assets can be erased from the account by accruing impairment losses ${ }^{[1]}$.

\section{Changing the equity to weaken the influence of related parties, and using unfair pricing of related parties to construct sales revenue}

At the beginning of its establishment, Shen Xian Yuan Wine Industry signed a wild mountain ginseng procurement contract with Shen Xian Yuan. Shen Xian Yuan needs to provide no more than 1 million wild ginseng to the Shen Xian Yuan Wine Industry every year, and the price of a ginseng is 800 yuan. Shen Xian Yuan said that wild ginseng has a longterm survival of more than fifteen years and is scarce, so it is preferentially supplied to the Shen Xian Yuan Wine Industry, resulting in a single supply of wild ginseng.

According to the SFC Punishment Decision, part of the equity of Shen Xian Yuan Wine Industry was sold to Peking University International Healthcare Co., Ltd. (hereinafter referred to as "PKU Healthcare") by Beijing Bishui Investment Co., Ltd. (hereinafter referred to as "Bishui Investment") in 2013-2014 and then bought it back.

During this period, Yu Chengbo took full control of the investment in Bishui Investment, while the general manager of Shen Xian Yuan was also taken by Yu Chengbo, the actual controller of Bishui Investment. Yu Chengbo still exerted a significant influence on Shen Xian Yuan Wine Industry. Other enterprises under the control, joint control or significant influence of key managers of the enterprise also form related parties with the enterprise according to the accounting standards of the enterprise. Therefore, even if Bishui Investment transferred $51 \%$ of the equity of Shen Xian Yuan Wine Industry in July 2013, Shen Xian Yuan and Shen Xian Yuan Wine Industry still constitute related parties during this period, and the transactions between the two are still related transactions.

The sales revenue was confirmed by the related party selling price of 800 yuan for the whole ginseng and 4,000 yuan for the broken ginseng per $\mathrm{kg}$. Since the wild ginseng sold by Shen Xian Yuan in the Shen Xian Yuan Wine Industry in 2013 was basically acquired from the intermediary, the price of related transactions is about twice of its purchase cost, so it is obvious that Shen Xian Yuan has utilized unfair related party selling price to inflate its income and profit.

The SFC stated that although Shen Xian Yuan claimed that all the wild ginseng sold were from its own production, in fact, most of them were purchased wild ginseng as mentioned above. Therefore, according to the market price of the purchase of wild ginseng, the annual sales of wild ginseng to the Shen Xian Yuan Wine Industry were recognized as $67,839,473$ yuan. Shen Xian Yuan used the related party's unfair pricing to increase its revenue by $73,729,327$ yuan, resulting in the inflated profit of $73,729,327$ yuan. In fact, the changes of sales revenue and sources in 2012-2013 can also reflect the signs of corporate fraud, as shown in table 1 .

TABLE I. SALES REVENUE AND SOURCE COMPOSITION FROM 2012 TO 2013

\begin{tabular}{|c|c|c|c|c|}
\hline Project & $\begin{array}{c}\text { Sales } \\
\text { revenue(100 } \\
\text { million yuan) }\end{array}$ & Major client & $\begin{array}{c}\text { Client } \\
\text { sales } \\
\text { revenue } \\
\text { share }\end{array}$ & $\begin{array}{c}\text { Source of } \\
\text { revenue }\end{array}$ \\
\hline 2012 & 0.75 & $\begin{array}{c}\text { Natural } \\
\text { Person }\end{array}$ & $23.63 \%$ & $\begin{array}{c}\text { Residential } \\
\text { sales; } \\
\text { Club income }\end{array}$ \\
\hline 2013 & 1.98 & $\begin{array}{c}\text { Shen Xian } \\
\text { Yuan Wine } \\
\text { Industry }\end{array}$ & $71.62 \%$ & $\begin{array}{c}\text { Wild ginseng; } \\
\text { Residential } \\
\text { sales; Club } \\
\text { income }\end{array}$ \\
\hline
\end{tabular}

From table 1, it can be seen that before 2013, the related transaction did not exist, and there was no phenomenon that the income was over-reliant on major customers. In 2013, there were three major changes in Shen Xian Yuan: Firstly, Shen Xian Yuan started to selling wild ginseng, and supplied wild ginseng to Shen Xian Yuan Wine Industry. This related transaction occurred. Secondly, Shen Xian Yuan turned a loss into a profit, with its annual operating income surging by $168 \%$, and its performance was very good. Thirdly, the sales of Shen Xian Yuan Wine Industry accounted for $71.62 \%$ of the operating revenue, so Shen Xian Yuan Wine Industry contributed $70 \%$ of the performance to Shen Xian Yuan in 2013.

In addition to the abnormal behaviors of Bishui Investment in 2013-2014 when its related party Shen Xian Yuan Wine Industry stock ownership was changed, it is easy to think that Shen Xian Yuan may have concealed unfair related party transactions. Obviously, in order to successfully pass the audit of the financial statements of NEEQ, the timing of Bishui Investment's choice to transfer and recover equity is too sensitive, and its means of fraud is not very good.

\section{ANALYSIS OF THE REASONS FOR SHEN XIAN YUAN 'S FINANCIAL FRAUD}

Bologua et al. ${ }^{[2]}$ put forward the theory of financial fraud GONE in 1993, believing that financial fraud consists of four factors: G (greed), O (opportunity), N (need) and E (exposure), which together determine the risk of corporate fraud. By using the GONE-theory to analyze the financial fraud case of Shen Xian Yuan, It is found from the source that the motivation of the NEEQ agricultural stock in Shen Xian Yuan ${ }^{[4]}$.

\section{A. Analysis of greed factors $(G)$}

The meaning of the greed factor here goes beyond the literal meaning, which can be understood as low morality, and bad values prevail, making financial fraud a behavior that conforms to its values. At the time of Shen Xian Yuan was listed, Yu Chengbo fully controlled the Bishui Investment. 
Therefore, Yu Chengbo and his wife are the actual controllers of Shen Xian Yuan.

$\mathrm{Yu}$ Chengbo is the chairman and former general manager of the company. The current general manager Li Dianwen and the financial director Zhao Dongying joined Beijing Bishui Manor Real Estate Development Co., Ltd. in 2005 and 1999 respectively and held important positions, which have been followed by $\mathrm{Yu}$ Chengbo. At that time, the main securities, Guojin Securities, also indicated the risk of actual controller and insufficient independence in the transfer statement. For example, the actual controller and the controlling shareholder all have large transactions. If the internal control system cannot be effective, the actual controller is capable of maximizing personal utility driven by bad values and instructing the current general manager and financial controller to implement financial fraud.

There are three situations in the company. The company's RMB 170 million loan for Bishui Investment, it provided guarantee to China Investment Guarantee Co., Ltd. and assumed joint guarantee responsibility; The company continued to make capital borrowing with related parties frequently; And the company is preparing to apply for a loan of 150 million yuan for Liaoning Shen Xian Yuan Biological Engineering Co., Ltd. Although there is no performance requirement for these NEEQ-listed companies, Yu Chengbo is likely to use relatively good performance and net asset valuation of Shen Xian Yuan to assist its related parties with personal interests to obtain bank funds and maintain the operation of related parties.

\section{B. Analysis of opportunity factors $(O)$}

The opportunity factor refers to the opportunity for potential fraudsters to have the power to commit fraud or to think that they will not be discovered and punished. Vulnerabilities in internal and external governance systems and their failure to implement them effectively enable potential fraudsters to have the right to fraud or to avoid the opportunity of fraudulent sights ${ }^{[5]}$.

Internal opportunity factor. Usually expressed as the shareholding structure, the staff allocation of the board of directors and the board of supervisors, as well as the completeness of the internal control system and the execution strength of internal control. $\mathrm{Yu}$ Chengbo and his wife indirectly controlled more than $80 \%$ of the shares of Shen Xian Yuan through Bishui Investment. The excessive equity concentration gives absolute control to $\mathrm{Yu}$ Chengbo and others, and the board of directors and the board of supervisors are directly and indirectly controlled by $\mathrm{Yu}$ Chengbo respectively, and one of the board members, Li Dianwen became the general manager and also followed Chengbo for many years. In fact, $\mathrm{Yu}$ Chengbo and others have combined their leadership and control rights, and his cronies are in important positions, quite a characteristic of family business. The actual controller risk prompted by Guojin Securities, the internal control degree defect and the internal control degree are not effectively implemented, can give potential fraudsters a chance.
External opportunity factor. Although the NEEQ has no requirements for performance, it is widely believed that these NEEQ-listed companies will not risk taking the act of fraudulent reporting. The auditing of financial reports by independent third-party accounting firms is supposed to be the last line of defense against financial fraud before the disclosure of financial reports. It is clear that the double standards of the SFC and the A-shares have reduced the auditing degree of auditing against the new NEEQ-listed company. Insufficient external auditing also undoubtedly provides an opportunity for financial fraud. In the case of Shen Xian Yuan, Beijing Xinghua accounting firm did not maintain the professional cautiousness of the agricultural stocks with low difficulty in making fraud, high identification difficulty and high audit risk ${ }^{[6]}$. Beijing Xinghua accounting firm and the main securities at that time did not obtain appropriate and sufficient audit evidence for the amount of wild ginseng's inventories and the number of excavations and related transactions, resulting in the construction of Shen Xian Yuan's fictional 129 million yuan, accounting for $116 \%$ of Shen Xian Yuan's disclosure of net profit after tax, escaped the attention of everyone. It was not until 2015 that the company's actions frequently caused the attention of the SFC. The negligence of the intermediary agencies when listing gives the opportunity of Shen Xian Yuan 's bold fraud.

\section{Analysis of need factors (N)}

The need factor, also known as the "motivation" factor, is the internal cause of financial fraud. Throughout the event, the shareholder of China's Agricultural Industry Development Fund Co., Ltd. (hereinafter referred to as "CFAD") have been operating frequently and are worth noting.

The capital increase agreement signed by Shen Xian Yuan and CFAD on Liaoning Bishui Forestry Development Co., Ltd. requires that the net assets of Shen Xian Yuan should not be less than 5 billion yuan. Jilin Huaxin Asset Price Evaluation Co., Ltd. assessed the total value of Shen Xian Yuan to reach 5.395 billion yuan in 2013. Yu Chengbo and others manipulated the 2013 performance, and the financial statements were greatly improved. In January 2014, Shen Xian Yuan received a capital increase of 1.24 million yuan through the Valuation Adjustment Mechanism with CFAD. In August, it increased its capital by 200 million yuan. The introduction of venture capital, instead of listing, gave Shen Xian Yuan great performance pressure, forcing $\mathrm{Yu}$ Chengbo and others to take risks for investment and stock value.

\section{Analysis of Exposure factors (E)}

The exposure factors include two meanings, one is the possibility of fraud discovery or disclosure; the second is the nature and degree of punishment for fraud.

The possibility of fraudulent behavior is generally determined by an independent third-party audit ${ }^{[7]}$. However, in the case of the Shen Xian Yuan fraud, the role of independent third-party auditing is very limited. The inventory of agricultural stocks is heavy in workload and difficult to be counted, and the inventory quantity is often calculated and estimated. Besides, the use of cash transactions in large quantities does not reflect the cost of sales costs. The financial 
statements after independent third-party audits are still low in accuracy. From this point of view, the fraudulent behavior of Shen Xian Yuan is less likely to be discovered. However, modern auditing has already summarized the audit risk of agricultural stocks. Using fictitious sales revenue for cash flow, we can still find signs of fraud in Shen Xian Yuan. From this perspective, Beijing Xinghua accounting firm did not maintain sufficient professional suspicion when auditing Shen Xian Yuan.

After the confession of Shen Xian Yuan's financial report was revealed, the SFC issued a fine of 600,000 yuan, one of the NEEQ-listed companies largest fines. The actual controller Yu Chengbo and general manager Li Dianwen were given a warning and each was fined 300,000 yuan; the financial controller Zhao Dongying was given a warning and a fine of 100,000 yuan; and other responsible persons were given a warning and a fine of 50,000 yuan. . However, it has not given any form of punishment to the relevant accounting firms and the main securities, which is easy to encourage intermediaries to ignore the signs of fraud in the financial statements for performance.

\section{SUMMARY}

Based on the factor analysis of Shen Xian Yuan's financial fraud under the GONE theory, it is shown that the experience and concept of financial fraud of A-share listed companies cannot be fully applied to the NEEQ-listed companies. As the first SFC punishment case in the NEEQ-listed companies, the appearance of Shen Xian Yuan was unexpected to many people. Simple and typical fraud practices, pure and obscure motives, can be described as simpler and more profound. Based on this, the following suggestions are proposed for the financial fraud management of the NEEQ-listed companies.

\section{A. Eliminating the old idea that the NEEQ-listed companies have insufficient motives for fraud}

Although the SFC has not set performance requirements for NEEQ, it still requires the company's financial statements to reflect the true financial status of the company in a fair way. However, most of the NEEQ-listed companies are small and medium-sized enterprises. The financial system and internal control system of the company before the listing are not perfect. The equity and management rights are more concentrated than the A-share listed companies. Therefore, their system is not well executed. What's more, the financial statements provided to banks, funds, customers and shareholders are inconsistent. However, from the time of decision to list on NEEQ, with higher regulatory and disclosure requirements, these companies have to sort out the bad debts, confuse accounts, cover up the ridiculous accounts, and neutralize the report data based on the different versions of statements and reality disclosed to the public, so as to smoothly pass the independent third-party audit and the review by the securities.

The small and medium-sized enterprises listed on NEEQ are more susceptible to the influence of past bad habits. In order to achieve a certain financing purpose, they will make financial statements fraud, it is necessary to spend a period of time on the disappearance of such inertia. It is even more necessary for the SFC to give more attention and constraints, promote the small and medium-sized enterprises improve the system of financial internal control, lower equity concentration, improve the credibility of financial statements.

\section{B. The risk of auditing agricultural stocks is too high, and} cautiously review the agricultural stocks whose performance has improved substantially in a short period of time

From the YINGUANGXIA event to the LANTIAN event, the fraud of agricultural stocks is characterized by typical practices, large amount of fraud, and high auditing difficulty. The A-share market is also very cautious about the introduction of agricultural stocks. For the NEEQ with loose thresholds, the agricultural stocks with malicious falsification cannot be allowed to enter the market, disrupting the order of the NEEQ market and dampening investors' confidence in the initial establishment of the NEEQ.

The SFC should continuously improve the regulations for the entry of the NEEQ into the market, eliminate the fraudulent atmosphere of the NEEQ, and provide a good environment for companies that are sincerely seeking development.

\section{Prior risk warning and limitation can better reflect the role of supervision than the investigation after the event}

Guojin Securities, the main securities company listed at that time, clearly indicated the operational risks, actual controllers and independent risks in the public transfer statement. At the time of listing review, the SFC should properly handle the risk warnings of independent third-party auditing, and should not be investigated until Shen Xian Yuan increased biological assets. The SFC's rigorous and pragmatic attitude is obvious to all. However, it cannot fully reflect the strong regulatory role of the SFC to issue an investigation decision until the evidence of the fraud is fully clear.

\section{REFERENCES}

[1] Wang Zhaolong. Shen Xian Yuan is the first financial fraud enterprise of the NEEQ[EB/OL]. Http://finance.qq.com/a/2016- 0604/011671.Htm. 2016-06-04.(In Chinese)

[2] Bologua G.J. Lindquist R.J. \& Wells J.T. Accoutant's Handbook of Fraud and Commercial Crime [J]. Jone Wiley \& Sons Inc, 1993 (5): 2081.

[3] Sean Humpherys. Kevin Moffitt. Mary Burns. William F. Feli. Identification of fraudulent financial statements using linguistic credibility analysis[J]. Decision Support Systems, 2011, 50(3): 585-594.

[4] Qin Jiangping. Accounting Fraud in Listed Companies: A Review and Enlightenment of Foreign Related Studies[J]. Accounting Research, 2005, (6): 69-73. (In Chinese)

[5] Wu Ge, Ye Chengang. Research on Characteristics of Financial Report Fraud: Empirical Data from A-share Listed Companies[J]. Audit Research, 2008(6): 34-41. (In Chinese)

[6] Lei Guangyong, Li Shufeng, Wang Xiujuan. Political Connection, Auditor Choice and Corporate Value [J]. Management World, 2009(7): 145-154. (In Chinese)

[7] Wei Lin, Xu Liwen, Liu Jia. Identification of Financial Report Fraud in Listed Companies: An Empirical Study Based on Triangle Theory[J]. Audit Research, 2011(2): 98-106. (In Chinese) 